\title{
Papers presented in the 7th Annual Meeting of the Society
}

1. M. Murakami (Institute of Statistical Mathematics): On the quantitative document analysis

2. Y. Ishibashi (Statlabo, Inc.), T. Udagawa (Hokkaido University of Education) and M. Iwasaki (Seikei University): A courseware for education of statistics

3. T. Tarumi (Okayama University) and T. Kataoka (Sanyo Gakuen Junior College): Statistical analysis using spread sheet

4. T. Tazawa (Mathematical System Institute Inc.): An object-oriented statistical analysis system "S-PLUS"

5. S. Asahara (SPSS Ltd.): Software for data analysis in GUI age "SPSS for Windows"

6. M. Hodono (Kyushu Kyoritsu University): A measure for warmth of heart

7. S. Yamaji (Institute for Nippon Private Education): An evalustion of learning structure chart based on characteristics of curriculum

8. K. Yanagi and T. Tarumi (Okayama University): Programming function of ML and its application

9. H. Douke (Kyushu Toukai University): A comparative study of sequential decision procedures on comparative clinical trials

10. S. Yamamoto and M. Goto (Shionogi Kaiseki Center): Performance of double power transformation for additivity

11. I. Tanaka (Government of Kanagawa Prefecture) and H. Onishi (University of Tsukuba): Factorial analysis of medical demand - a case study of Kanagawa Prefecture

12. Y. Mori (Kurashiki Municipal Junior College): Variable selection procedure in principal component analysis using RV coefficient

13. M. Mizuta (Hokkaido University): Fitting curves and surfaces to multivariate data

14. O. Miyatake, Y. Yoshizawa (College of Industry Technology) and M. Ichimura (Okayama University of Science): Arrangement of experimental data with systematic errors

15. H. Imai and Y. Sato (Hokkaido University): Correspondence analysis and uniformity of a contingency table

16. M. Fujiwara (NEC): Mechanism of auto-organization of brain-nerve system of organs

17. Z. F. Mateo (Okayama University), Y. Odaka (Okayama University of Science) and Y. Tanaka (Okayama University): Sensitivity analysis in factor analysis regression: A numerical study

18. S. Watadani (Sanyo Gakuen Junior College) and Y. Tanaka (Okayama University): Software for sensitivity analysis of covariance structure analysis - Factor analysis version SACS/FA 
19. K. Inoue (NEC Software Okayama Ltd.) and Y. Tanaka (Okayama University): Software for sensitivity analysis of factor analysis -development of SAF/S using S-language(II)

\section{Papers presented in the 7th Symposium of the Society}

1. Y. Ishibashi (Statlab. Inc.): Courseware for statistics, Part2

2. M. Hodono (Kyushu Kyoritsu University): HODONO MAKOTO system for assessment of educational attainments and AHO assessment method

3. O. Miyatake, Y. Yoshizawa (Collage of Industrial Technology), M. Toyama and M. Ichimura (Okayama University of Science): Unification of measurements involving systematic errors

4. K. Matsuo (Fujitsu Laboratory): Genetic algorithm and artificial life

5. K. Hiramatsu (Governor of Oita Prefecture): Movement from enriched "for one village one atomosphere" campaign to a happy provence network

6. S. Shirahata (Osaka University): Interval estimation of population mean based on ranked set sampling

7. A. Kudo, K. Ujiie and Y. Yamamoto (Toukai University): Computation and application of orthant probability

8. M. Kuroda and M. Ichimura (Okayama University of Science): Unification of measurements involving systematic errors

9. T. Nagai (Oita University): Bootstrap approaches to time series analysis

10. T. Ueda (Mitsubishi Computer Systems Ltd.): Efficient use of S-PLUS

11. H. Yoshida, S. Kyoda, H. Kimura (Okayama University of Science) and C. Asano (Souka University): Development of Micro-NISAN system with MS-Windows

12. S. Watadani (Sanyo Gakuen Junior College) and Y. Tanaka (Okayama University): A statistical software for sensitivity analysis of covariance structure model "SACS(SEM)"

13. H. Eto and S. Iwagami (Fujitsu Oita Software Lab. Ltd.): Information sharing between SGML-DB and Tex

14. M. Mizuta (Hokkaido University): Dynamic scatterplot matrix and additional functions

15. Y. Fukuhara, S. Nakagawa and M. Ichimura (Okayama University of Science): Asymptotic distribution of $\sqrt{b}$, from a non-normal population

16. S. Onishi, M. Kawaguchi (Hokkai-Gakuen University) and H. Imai (Hokkaido University): A clustering algorithm based on the test of homogeneity for contingency table 
17. S. Yamamoto, K. Kuribayashi and M. Goto (Shionogi Kaiseki Center): Exact fitting and performance of binary regeression model

18. Y. Ochi and T. Obata (Oita University): Regression diagnostics for multinomial responses

19. A. Sakuma (Tokyo Medical and Dental University): Randomized controlled trials

20. T. Akazawa and K. Inoue (NEC Software Okayama Ltd.) and T. Tarumi (Okayama University): Representation of statistical analysis knowledge on check sheets

21. K. Funada (Fujitsu Tokyo Systems Ltd.): An approach to "GCP-support-System" with tools: IP and ODB II

22. M. Kimura, M. Kuroda, H. Kimura, S. Nakagawa, M. Ichimura and S. Kadowaki (Okayama University of Science): Development and application of a curriculum support system

23. S. Kan (Syakai Jyouhou Service Ltd.): A proposed rule for offering data and free use of our data

24. H. Kubo and H. Hirooka (Yamanouchi Pharmaceutical Co. Ltd.): Auto-coding in clinical data processing

25. K. Yanagi and T. Tarumi (Okayama University): An object representation of knowledge data base in statistical analysis

26. T. Tasaki (Shionogi Kaiseki Center): The forefront in statistical computing 